\title{
Review of: "Microbial methylation potential of mercury sulfide particles dictated by surface structure"
}

\author{
Jean Remy Guimaraes ${ }^{1}$ \\ 1 Universidade Federal do Rio de Janeiro
}

Potential competing interests: The author(s) declared that no potential competing interests exist.

The text by Tian et al. reveals a significant and refreshing mindset change when its first sentence says “Methylmercury $(\mathrm{MeHg})$ production is primarily mediated by anaerobic microorganisms in natural aquatic environments". This is notable, as for decades we have been used to read that MeHg production occurs preferentially in anaerobic environments, which is definitely not the same thing. This mantra, or trap of linear thought, made an amalgam between anaerobes and their typical environment, badly hindering $\mathrm{Hg}$ research, as it took years to show that anaerobes are alive and well, and methylating $\mathrm{Hg}$, in apparently oxic environments. The main issue here was scale. Either because of this logical trap or because of technical limitations, we have overlooked for years the vast world of Hg-relevant micro and nano scale microbiological and chemical processes, and more important than that, their interactions.

The issue of the role of nanocinnabar compounds in the bioavailability of $\mathrm{Hg}$ for methylation has been around for some time, but no research group so far had been so deep in the study of the interaction between microbial $\mathrm{Hg}$ methylation and nanocinnabar formation and structure, and to pinpoint the mechanistic of this interaction and its singular dependency on the specific nanocinnabar (111) facet. A real check-mate.

The authors seem fully conscious of the breakthrough when they write that their study "paves the way toward incorporating particulate phases and interfacial processes into predictive models of MeHg production". Modellers will be happy, their models will have much better chances to describe the cycle of this elusive element, other than in very site-specific conditions.

Though the authors explore very well the consequences of their findings to the issue - among many others - of "old" versus "new" Hg, so relevant to management of $\mathrm{Hg}$ in the environment, the importance of their notable study goes well beyond the field of $\mathrm{Hg}$ biogeochemistry and the authors are clear about the importance of their findings to studies on the cycle of other important elements, toxic or not. I also wish to congratulate the authors for the excellent graphic choices, that synthetized very clearly an amazing body of complex data.

The study illustrates magisterially how a multi-disciplinary approach, combined with state-of-the-art methods, is essential to address or manage complex environmental issues.

I foresee that this work will produce stimulating shock waves in biogeochemistry and will be heavily cited. 
